\title{
VGLL4 functions as a new tumor suppressor in lung cancer by negatively regulating the YAP-TEAD transcriptional complex
}

Wenjing Zhang ${ }^{1, *}$, Yijun Gao ${ }^{1, *}$, Peixue $\mathrm{Li}^{1,{ }^{*}}$, Zhubing Shi ${ }^{1}$, Tong Guo ${ }^{1}$, Fei Li ${ }^{1}$, Xiangkun Han ${ }^{1}$, Yan Feng ${ }^{1}$, Chao Zheng ${ }^{1}$, Zuoyun Wang ${ }^{1}$, Fuming $\mathrm{Li}^{1}$, Haiquan Chen ${ }^{2,3}$, Zhaocai Zhou ${ }^{1}$, Lei Zhang ${ }^{1}$, Hongbin $\mathrm{Ji}^{1}$

${ }^{I}$ State Key Laboratory of Cell Biology, Institute of Biochemistry and Cell Biology, Shanghai Institutes for Biological Sciences, Chinese Academy of Sciences, Yueyang Road, Shanghai 200031, China; ${ }^{2}$ Department of Thoracic Surgery, Fudan University Shanghai Cancer Center, Shanghai 200032, China; ${ }^{3}$ Department of Oncology, Shanghai Medical College, Fudan University, Shanghai 200032, China

Lung cancer is one of the most devastating diseases worldwide with high incidence and mortality. Hippo (Hpo) pathway is a conserved regulator of organ size in both Drosophila and mammals. Emerging evidence has suggested the significance of Hpo pathway in cancer development. In this study, we identify VGLL4 as a novel tumor suppressor in lung carcinogenesis through negatively regulating the formation of YAP-TEAD complex, the core component of Hpo pathway. Our data show that VGLL4 is frequently observed to be lowly expressed in both mouse and human lung cancer specimens. Ectopic expression of VGLL4 significantly suppresses the growth of lung cancer cells in vitro. More importantly, VGLL4 significantly inhibits lung cancer progression in de novo mouse model. We further find that VGLL4 inhibits the activity of the YAP-TEAD transcriptional complex. Our data show that VGLL4 directly competes with YAP in binding to TEADs and executes its growth-inhibitory function through two TDU domains. Collectively, our study demonstrates that VGLL4 is a novel tumor suppressor for lung cancer through negatively regulating the YAP-TEAD complex formation and thus the Hpo pathway.

Keywords: VGLL4; lung cancer; Hippo pathway; YAP

Cell Research (2014) 24:331-343. doi:10.1038/cr.2014.10; published online 24 January 2014

\section{Introduction}

Lung cancer is one of the most prevalently diagnosed cancer types with high mortality worldwide [1]. From pathological standpoints, lung cancer can be roughly classified into two major subtypes: non-small cell lung cancer (NSCLC) and small cell lung cancer (SCLC). Featured as the most common type, NSCLC that accounts for $80 \%$ of all cases can be further sub-divided into adenocarcinoma (ADC, $\sim 48 \%$ ), squamous cell

\footnotetext{
*These three authors contributed equally to this work.

Correspondence: Hongbin $\mathrm{Ji}^{\mathrm{a}}$, Lei Zhang ${ }^{\mathrm{b}}$, Zhaocai Zhou ${ }^{\mathrm{c}}$

${ }^{a}$ E-mail: hbji@sibcb.ac.cn

${ }^{\mathrm{b}}$ E-mail: RayZhang@sibcb.ac.cn

'E-mail: zczhou@sibcb.ac.cn

Received 17 June 2013; revised 13 August 2013; accepted 11 September 2013; published online 24 January 2014
}

carcinoma (SCC, $\sim 28 \%$ ) and large cell carcinoma (LCC, $\sim 24 \%$ ) $[2,3]$. Despite the great advances achieved in recent targeted therapies, lung cancer still holds a quite poor prognosis and its 5 year survival rate remains as low as $10 \%-15 \%$ [4].

Hippo (Hpo) pathway is a newly discovered signaling cascade, which is initially considered as a key regulator of tissue growth and controls organ size via regulating the YAP-TEAD complex [5-7]. In recent years, accumulating evidence has suggested that Hpo pathway also plays an important role in cancer development. Inactivation of upstream members in Hpo pathway either by mutations or epigenetic silencing have been reported in human epithelial cancers [8]. Moreover, YAP, the essential downstream effector of Hpo pathway, is frequently highly expressed in a wide spectrum of human solid tumors and significantly associated with poor clinical outcomes [9-12]. Functional studies show that ectopic 
YAP expression is sufficient to drive cell proliferation, transformation, invasion and epithelial-mesenchymal transition (EMT) [13]. Activation of YAP can drive the expansion of epidermal stem cell compartment as well as the formation of SCC-like tumors in mouse skin [14]. Liver-specific activation of YAP either by transgene overexpression or $M s t 1 / 2$ deletion results in a dramatic increase in liver size and the development of liver tumor [15-17].

Deregulation of Hpo pathway leads to the stabilization and nuclear sequestration of YAP. Nuclear YAP binds to and activates the transcription factors TEADs (the TEAD/TEF family transcription factors) and eventually turns on the expression of downstream genes including CTGF and CYR61 [5]. The mammalian genome contains four highly homologous TEAD/TEF family members (TEAD1-4), which are expressed in diverse tissues from pre-implantation embryos to adult tissues [18]. Previous studies have shown that TEADs are also involved in human cancers. High TEAD1 expression correlates with poor clinical outcome in prostate cancer [19]. Amplification and high expression of TEAD4 have been found in serous fallopian tube carcinoma, basal breast cancer and testicular germ cell tumors [20-22]. Experimentally, knockdown of TEAD1 reduced cell proliferation and impaired acinar formation ability [19]. TEAD4 alone also promoted anchorage-independent growth in MCF10A cells [23]. These observations indicate that TEADs may also play an important role in human cancers.

Vestigial-like (VGLL) proteins have recently emerged as a new group of TEAD-interacting partners participating in tumorigenesis. VGLL proteins are transcriptional cofactors that are named after Drosophila transcriptional co-activator Vestigial $(\mathrm{Vg})$, the master regulator of wing development. There are 4 VGLL proteins in mammals, named VGLL1-4. These proteins bear no sequence similarity except for the TDU domain (the TEAD-interacting domain) [24-28]. Previous studies showed that VGLL1 promotes cell proliferation and exhibits high expression in basal-like breast cancer $[29,30]$. Similarly, $V G L L 3$ is amplified in soft tissue sarcoma and inhibition of VGLL3 results in decreased cell proliferation and migration [31]. Different from other members in VGLL family, VGLL4 contains an extra TDU domain and is considered to be functionally different. For example, VGLL4 can promote apoptosis via negatively regulating inhibitor of apoptosis proteins (IAPs) [32]. However, the exact role of VGLL4 in cancers, especially in lung cancer, and whether and how VGLL4 is involved in the Hpo pathway are not clear.

Here, we found that VGLL4 is consistently downregu- lated in both murine and human lung ADC specimens. Our data further proved that VGLL4 functions as a suppressor of lung cancer growth and progression via direct competition with YAP in forming the complex with TEADs through two TDU domains.

\section{Results}

VGLL4 is downregulated in both mouse and human lung ADC specimens

To study the potential role of VGLL4 in lung cancer, we first examined the expression level of VGLL4 in $\operatorname{Kras}^{\mathrm{G} 12 \mathrm{D}}$-based lung cancer mouse model. Through quantitative RT-PCR analysis, we found that murine lung ADCs derived from $\mathrm{Kras}^{\mathrm{G} 12 \mathrm{D}}$ model expressed lower level of Vgll4 mRNA relative to mouse normal lungs (Figure 1A). Through immunohistochemistry (IHC) study, we further showed that VGLL4 protein levels decreased in mouse lung ADCs (Figure 1B). Moreover, VGLL4 displayed a more diffused cytoplasmic staining in lung ADCs, but a predominant nuclear staining was seen in normal lungs (Figure 1B).

We further examined the expression status of VGLL4 in human lung ADC specimens. Interestingly, we found that almost all the human lung ADC samples (29 of 30) had a relatively lower VGLL4 expression in comparison with paired pathologically normal lungs (Figure 1C). IHC studies in a cohort containing 27 normal lungs and 77 lung ADCs showed that $92.6 \%$ of patients ( 25 out of 27 ) exhibited high nuclear VGLL4 expression in their normal lungs, whereas only $22.1 \%$ of patients (17 out of 77) had high nuclear expression of VGLL4 in their lung ADCs. The pattern of VGLL4 nuclear expression was statistically different between normal lungs and lung ADCs $(P<0.01)$ (Figure 1D and 1E). Taken together, these results indicated that VGLL4 is consistently expressed at lower level in lung ADC compared with normal lung tissues.

\section{VGLL4 suppresses lung tumor cell growth in vitro}

We next investigated the potential biological function of VGLL4 in lung cancer. We first examined the expression of VGLL4 in a series of lung cancer cell lines and found that A549 and CRL-5872 had relatively low but detectable level of VGLL4 expression (Supplementary information, Figure S1). We then overexpressed VGLL4 in these two cell lines. Our data showed that ectopic VGLL4 expression significantly inhibited the proliferation of these two cell lines (Figure 2A and 2B and Supplementary information, Figure S2). Cell cycle analysis showed that both A549 and CRL-5872 cells with VGLL4 overexpression displayed a reduced cell ratio in $\mathrm{G} 2 /$ 
A

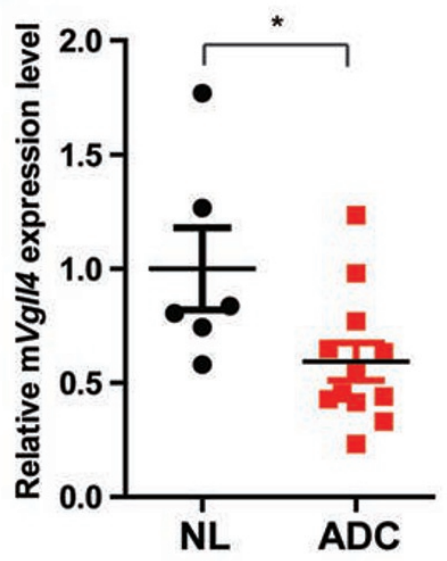

B

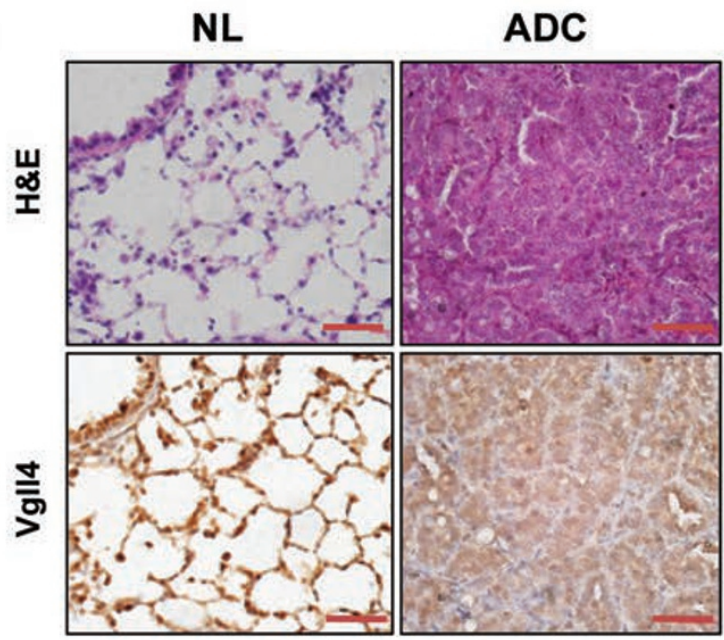

C
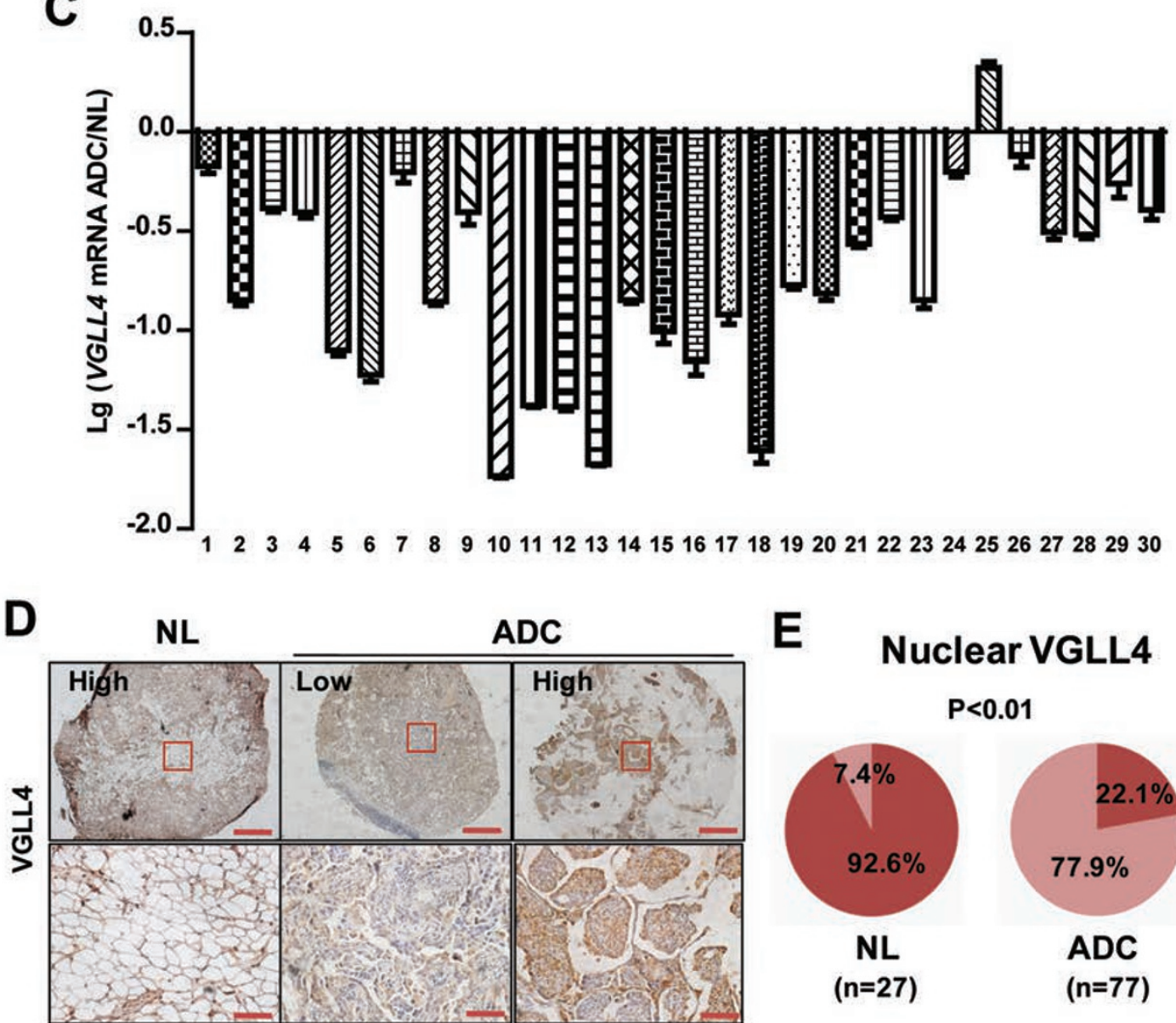

E Nuclear VGLL4 $P<0.01$

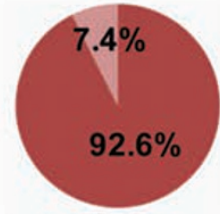

NL $(n=27)$

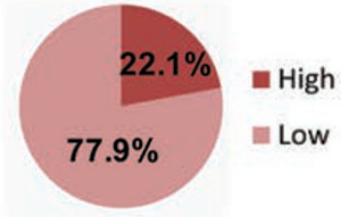

ADC

( $n=77$ )

Figure 1 VGLL4 is lowly expressed in both human and mouse lung adenocarcinomas. (A) Real-time PCR quantification of Vgll4 mRNA levels in mouse Kras $^{\mathrm{G} 12 \mathrm{D}}$ lung adenocarcinoma and normal lungs. Data are shown as means $\pm \mathrm{SEM}$. ${ }^{*} P<0.05$. (B) H\&E and Vgll4 immunohistochemical staining on mouse Kras ${ }^{\mathrm{G} 2 \mathrm{D}}$ lung adenocarcinoma and normal lungs. Scale bar, $50 \mu \mathrm{m}$. (C) Quantification of VGLL4 mRNA levels in human lung adenocarcinoma and paired pathologically normal lungs by real-time PCR. The values were presented as log10 ratio of the VGLL4 expression of human lung adenocarcinomas vs normal lung specimens. (D) Immunohistochemical staining of VGLL4 on human lung adenocarcinoma and normal lungs. Scale bar, 500 $\mu \mathrm{m}$ (top) and $50 \mu \mathrm{m}$ (bottom). (E) Statistical analysis of nuclear VGLL4 staining in human lung adenocarcinoma and normal lung specimens. ADC, adenocarcinoma; NL, normal lung. 
A

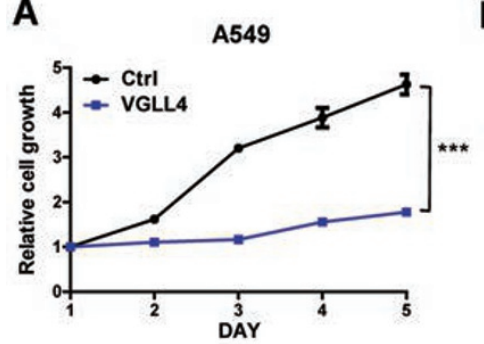

E

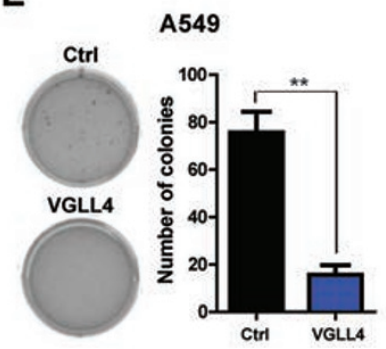

B

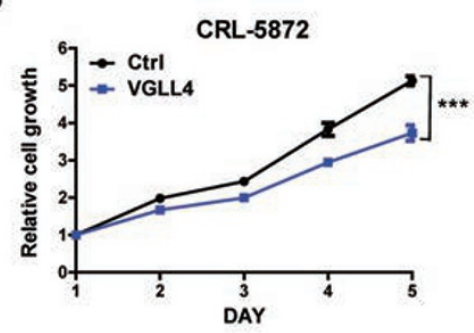

$\mathbf{F}$

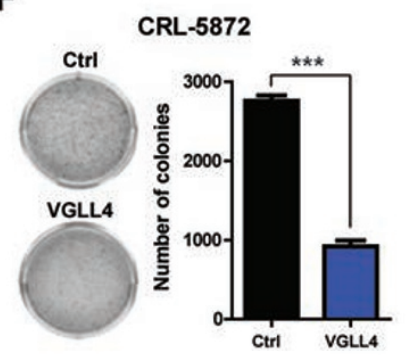

C

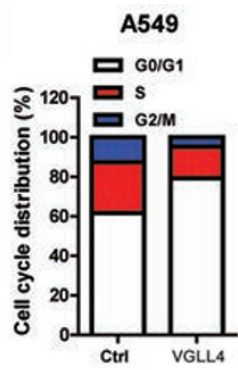

G

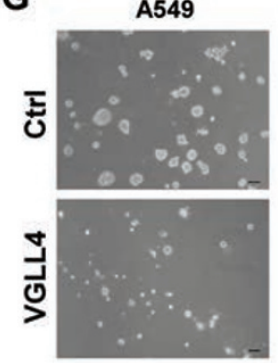

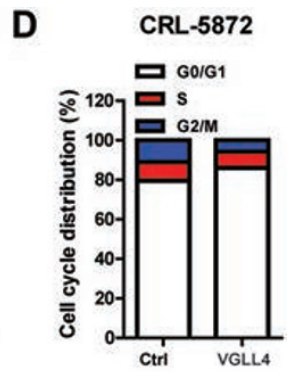

H

H $\quad$ CRL-5872

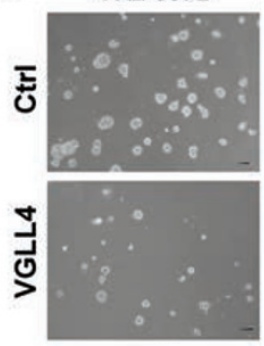

Figure 2 VGLL4 inhibits lung cancer cell growth in vitro. (A and B) The proliferations of A549 (A) and CRL-5872 (B) cells with or without ectopic VGLL4 expression were detected by MTT assay. Data are shown as means \pm SEM. ${ }^{* * *} P<0.005$. (C and D) Cell cycle distributions of A549 (C) and CRL-5872 (D) cells with or without VGLL4 overexpression were assessed by FACS assay. (E and F) Colony formation abilities of A549 (E) and CRL-5872 (F) cells with or without ectopic VGLL4 expression were assessed by soft agar assay. Data are shown as means \pm SEM. ${ }^{* *} P<0.01$; ${ }^{* * *} P<0.005$. (G and H) A549 (G) and CRL$5872(\mathrm{H})$ cells with or without VGLL4 overexpression were cultured in Matrigel for 7 days. Colony size was shown by photos. Scale bar, $0.01 \mathrm{~mm}$.

$\mathrm{M}$ phase (Figure 2C and 2D). We further showed that VGLL4 overexpression inhibited both the anchorageindependent cell growth in soft agar (Figure 2E and 2F) and colony proliferation in Matrigel culture of A549 and CRL-5872 cells (Figure $2 \mathrm{G}$ and $2 \mathrm{H}$ ). These data clearly demonstrated that VGLL4 negatively regulates lung cancer cell proliferation and growth in vitro.

VGLL4 suppresses lung cancer progression in de novo mouse model

We next asked whether VGLL4 plays a tumor suppressive function in vivo. To address this, we employed a $\mathrm{Kras}^{\mathrm{G} 12 \mathrm{D}}$-based de novo lung cancer mouse model. We first examined the Vgll4 expression in lung tumors derived from either $\operatorname{Kras}^{\mathrm{G} 12 \mathrm{D}}$ or $p 53^{\mathrm{L} / \mathrm{L}} / \operatorname{Kras}^{\mathrm{G} 12 \mathrm{D}}$ or $L k b 1^{\mathrm{L} /}$ ${ }^{\mathrm{L}} / \operatorname{Kras}^{\mathrm{G} 12 \mathrm{D}}$ mice. Our data showed that the lung tumors from $L k b 1^{\mathrm{L} / \mathrm{L}} / \mathrm{Kras}^{\mathrm{G} 12 \mathrm{D}}$ mice exhibited the lowest $\mathrm{Vgll4}$ expression (Supplementary information, Figure S3). We then decided to use $L k b 1^{\mathrm{L} / \mathrm{L}} / \mathrm{Kras}^{\mathrm{G} 12 \mathrm{D}}$ mice to verify the in vivo function of VGLL4. We delivered the lentivirus carrying either Cre (Ctrl-Cre) or VGLL4-Cre to $L k b 1^{\mathrm{L} /}$ ${ }^{\mathrm{L}} /$ Kras $^{\mathrm{G} 12 \mathrm{D}}$ mice through nasal inhalation as previously described [33]. We then analyzed the mice at 13 weeks post viral administration for gross inspection and patho- logical study (Figure 3A). We confirmed the VGLL4 expression in lung tumors via immunostainning (Figure $3 \mathrm{~B})$. Our data showed that the ectopic expression of VGLL4 did not cause a significant change in the total tumor number and tumor area despite a detectable trend that mice infected with VGLL4-Cre harbored less tumor area (Supplementary information, Figure S4). However, detailed pathological analysis revealed that the percentage of large tumors $\left(\geq 0.5 \mathrm{~mm}^{2}\right)$ was significantly lower in VGLL4-Cre group (Figure 3C and 3D). Consistently, a lower proliferation rate indicated by Ki-67-positive staining was observed in lung tumors with VGLL4 overexpression (Figure 3E and 3F). Moreover, the percentage of high-grade tumors was slightly decreased in VGLL4-Cre group in comparison with control group (Supplementary information, Figure S5). Taken together, these data suggested that VGLL4 may function as a negative regulator of lung tumor progression in vivo.

\section{TEADs mediate VGLL4's suppression on lung tumor cell growth \\ Previous study showed that VGLL4 physically inter- acts with TEAD1 [24]. As mentioned above, TEAD fam- ily contains four highly homologous proteins TEAD1-4.}


Among them, TEAD4 is highly expressed in the lung. We therefore examined the potential interaction of VGLL4 and TEAD4. Our data showed that VGLL4 and TEAD4 colocalized in the nucleus and had strong binding with each other (Supplementary information, Figure S6). We then asked whether TEADs are important for VGLL4's suppressive role in lung cancer cell growth. We found that VGLL4 no longer exhibited a suppressive function when TEADs were inactivated through TEAD1/3/4 RNAi (Supplementary information, Figure S7). Previous studies using mammalian two-hybrid system have indicated that deletion of both TDU domains in VGLL4 prevents its interaction with TEAD1 [24]. To confirm the importance of these TDU domains in VGLL4-TEAD4 interaction, we performed co-immunoprecipitation experiments and found that deletion of both TDU domains abolished the interaction between VGLL4 and TEAD4 (Figure 4A). To further prove our hypothesis, we used a VGLL4 mutant form (VGLL4 with the deletion of both TDU domains) to see whether VGLL4-TEAD interaction is required for VGLL4's inhibitory role in tumor cell growth. Our data revealed that deletion of both TDU domains significantly attenuated the inhibitory function of VGLL4 in A549 cell growth in 2D culture and colony formation in soft agar (Figure 4B and 4D and Supplementary information, Figure S8A). Similar results were obtained in another lung cancer cell line CRL-5872 (Figure $4 \mathrm{C}$ and $4 \mathrm{E}$ and Supplementary information, Figure S8B). Thus, these findings suggested that TEADs mediates the inhibitory function of VGLL4 in lung cancer cell growth.

\section{VGLL4 inhibits the transcriptional activity of TEADs}

Increased TEAD expression has been observed in human cancers [27]. Also, TEADs have been reported to facilitate the growth of certain cell types [19, 23]. As transcriptional factors, TEADs regulate a number of growthpromoting genes. In consideration of this, we asked whether VGLL4 modulates the transcriptional activity of TEADs. We took advantage of an established TEAD4responsive element-driven luciferase reporter system [34] and found that ectopic VGLL4 expression significantly reduced the TEAD4-dependent luciferase activity (Figure 5A). In contrast, the VGLL4 mutant without two TDU domains obviously lost its inhibitory function. We further examined whether VGLL4 altered the TEAD transcrip-
A
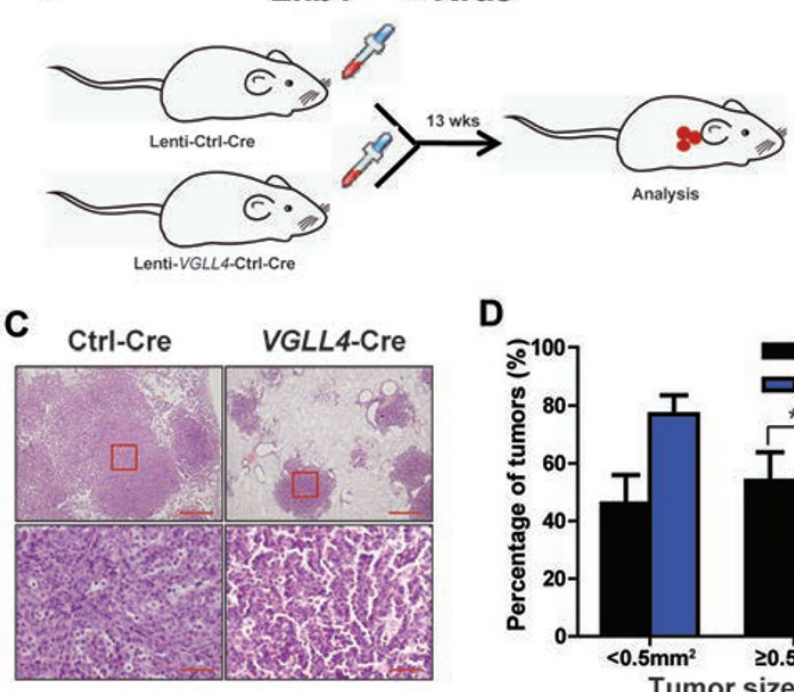

D

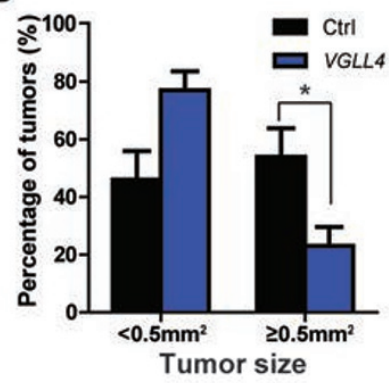

B
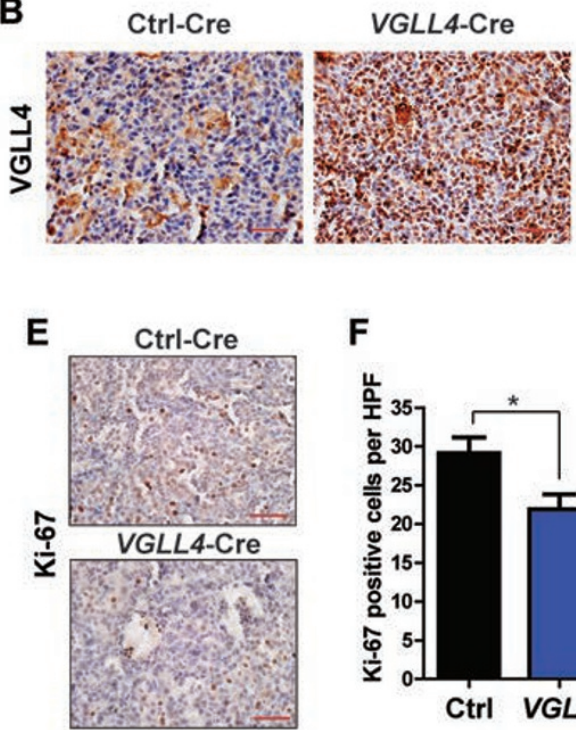

$\mathbf{F}$

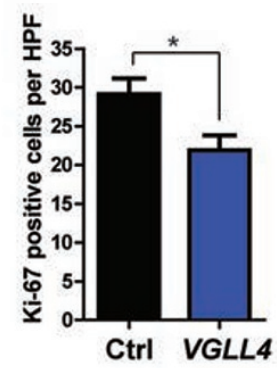

Figure 3 VGLL4 significantly suppresses lung cancer progression in de novo mouse model. (A) Scheme of viral infection

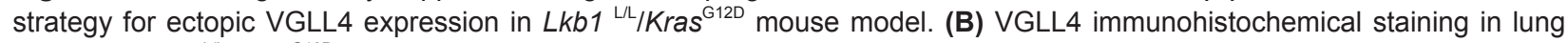
tumors of $L k b 1^{L / L} / K r a s^{G 12 D}$ mouse at 13 weeks post viral infection with Lenti-Cre or Lenti-VGLL4-Cre. Scale bar, $50 \mu \mathrm{m}$. (C) Representative H\&E photos of $L k b 1^{L / L} / K_{r a s}{ }^{G 12 D}$ mice lung at 13 weeks post viral infection with Lenti-Ctrl-Cre or Lenti-VGLL4Cre. Scale bar, $500 \mu \mathrm{m}$ (top) and $50 \mu \mathrm{m}$ (bottom). (D) Statistical analysis of lung tumors with the indicated size from $L k b 1^{1 / L} /$ Kras $^{\mathrm{G} 12 \mathrm{D}}$ mice virally infected with Lenti-Ctrl-Cre or Lenti-VGLL4-Cre. Data are shown as means $\pm \mathrm{SEM}$. ${ }^{\star} P<0.05$. (E) Ki-67 immunohistochemical staining of lung sections from $L k b 1^{L / L} / K r a s^{G 12 D}$ mice virally infected with Lenti-Ctrl-Cre or Lenti-VGLL4Cre. Scale bar, $50 \mu \mathrm{m}$. (F) Quantitative analyses of proliferative index in lung tumors from $L k b 1^{L / L} / K \mathrm{Kas}^{\mathrm{G} 12 \mathrm{D}}$ mice virally infected with Lenti-Ctrl-Cre or Lenti-VGLL4-Cre. Data are shown as means \pm SEM. ${ }^{*} P<0.05$. 


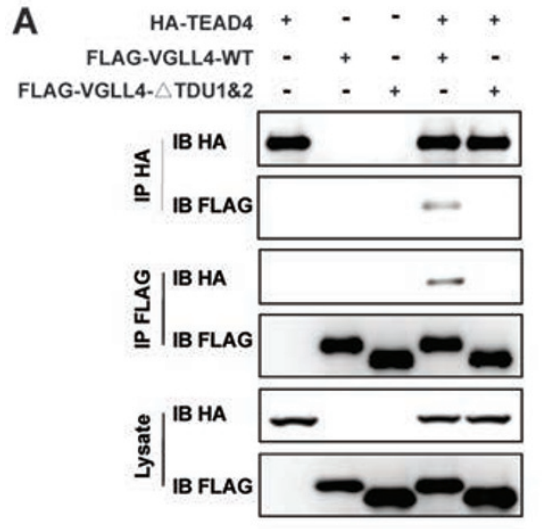

B

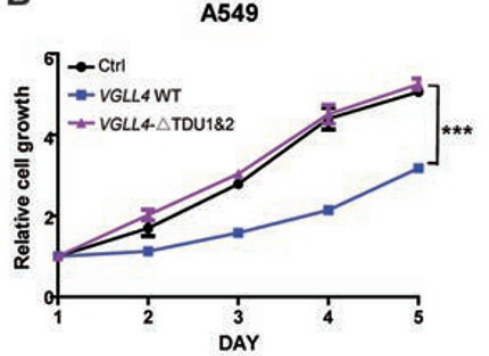

C

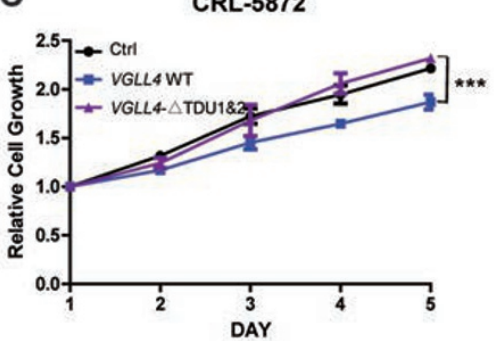

D

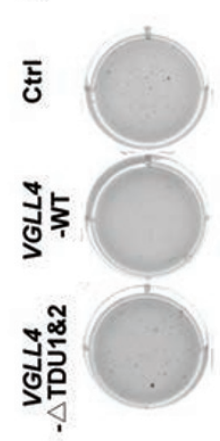

A549

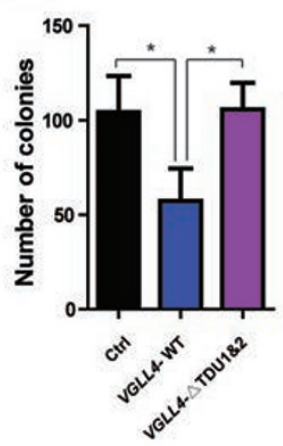

E

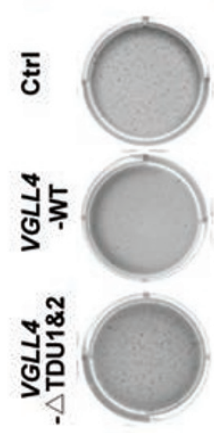

CRL-5872

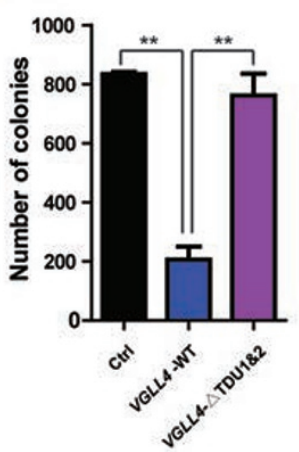

Figure 4 TEADs mediate VGLL4's inhibitory role in lung tumor cell growth. (A) Co-immunoprecipitation experiments of TEAD4 with wild-type (WT) VGLL4 or VGLL4 mutant with deletion of two TDU domains in HEK-293T cells. TEAD4 is HAtagged and VGLL4 is FLAG-tagged. (B and C) Deletion of two TDU domains abolishes the inhibitory role of VGLL4 in the cell proliferation of human lung cancer cell lines A549 (B) and CRL-5872 (C). Data are shown as means \pm SEM. ${ }^{* *} P<0.005$. (D and E) Deletion of two TDU domains abolishes the inhibitory role of VGLL4 in the colony formation of human lung cancer cell lines A549 (D) and CRL-5872 (E) in soft agar. ${ }^{*} P<0.05$; ${ }^{*} P<0.01$.

tion downstream genes [13, 35]. Our data showed that ectopic expression of VGLL4, but not VGLL4 lacking two TDU domains decreased the mRNA levels of two well-known downstream target genes $C T G F$ and $C Y R 61$ in HEK-293T and A549 cells, respectively (Figure 5B and 5C). Consistently, VGLL4 knockdown significantly increased TEADs' transcriptional activity in HEK-293T (Figure 5D and Supplementary information, Figure S9) as well as the CTGF and CYR61 mRNA levels in human bronchial epithelial cells (Beas2B) and CRL-5807 cells (Figure 5E and 5F and Supplementary information, Figure S10). Together, these data identified VGLL4 as a negative regulator of TEADs' transcriptional activity.

VGLL4 inhibits the function of YAP-TEAD transcriptional complex through direct competition

TEADs require transcriptional cofactors to fully activate the downstream gene transcription. YAP has been well documented as a major transcriptional co- activator of TEADs through direct binding to TEADs and facilitates downstream target gene expression [13]. We therefore asked whether VGLL4 suppresses TEADs' transcriptional activity by attenuating YAP-induced activation of TEADs. Through luciferase reporter assay, we found that overexpression of YAP enhanced the activity of TEAD4-responsive luciferase reporter, whereas VGLL4 and YAP co-expression led to a dramatic decrease of TEAD4-responsive luciferase reporter activity (Figure 6A). Similar results were observed using another luciferase reporter driven by the promoter of CTGF (Supplementary information, Figure S11). We further analyzed TEAD downstream target gene expression by quantitative RT-PCR. Overexpression of YAP activated the transcription of CTGF and CYR61. However, the mRNA levels of these two genes were downregulated sharply when YAP and VGLL4 were co-expressed in HEK-293T and A549 cells (Figure 6B and 6C).

As VGLL4 inhibited TEADs' transcriptional activity 
enhanced by YAP, we then investigated the underlying mechanism. As our data showed, unlike currently known mechanisms, ectopic expression of VGLL4 neither decreased the YAP protein level nor led to YAP cytoplasmic sequestration (Supplementary information, Figure S12). Because of the predominant nuclear location of VGLL4, we wondered whether it is possible that VGLL4 competes with YAP for binding to TEADs. To answer this, MBP-fused YAP coupled on amylose resin was mixed with TEAD4 in the presence of different amounts of VGLL4. The dosage-dependent pull-down assay clearly showed a decreasing binding of TEAD4 to YAP when increasing amounts of VGLL4 protein were added (Figure 6D). Therefore, our observations supported the notion that VGLL4 suppresses TEADs' activity through competing with YAP for TEAD binding. YAP had been reported to promote cell growth in several types of tumor cells $[10,12,36]$. Therefore, we then investigated whether VGLL4 can abrogate YAP's promotive effect on tumor cell growth. We found that ectopic expression of YAP facilitated A549 cell proliferation and enhanced its colony formation ability, whereas VGLL4 co-expression abolished this effect (Figure 6E and 6F and Supplementary information, Figure S13). Thus, the above data indicated that VGLL4 can diminish YAP-enhanced TEADs' transcriptional activity and YAP-promoted tumor cell growth by impairing YAP-TEAD interaction.
A

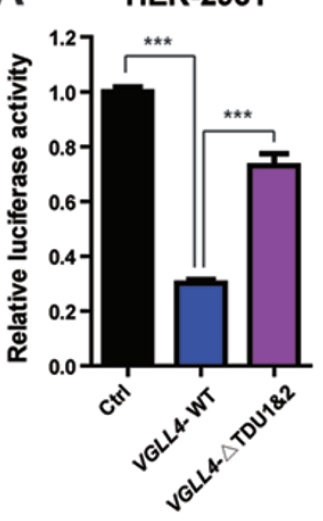

D

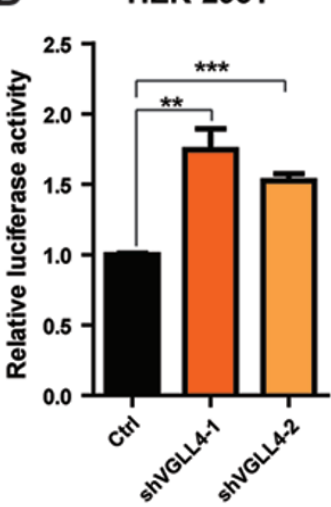

B

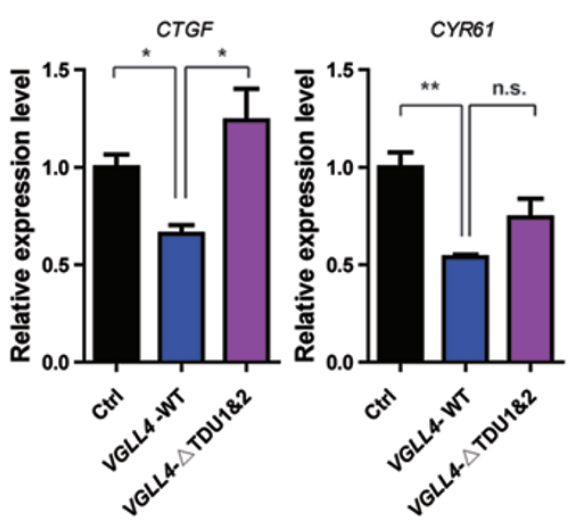

$\mathbf{E}$

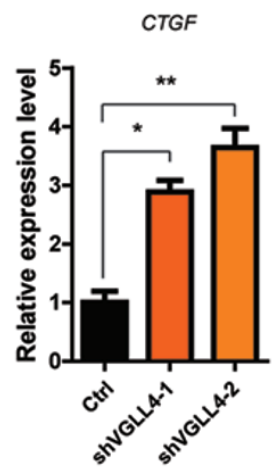

Beas2B

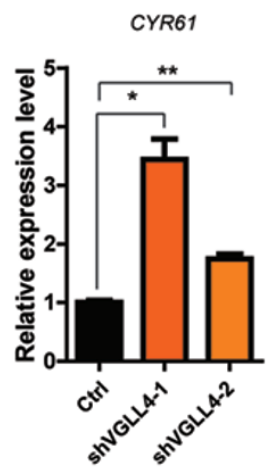

C

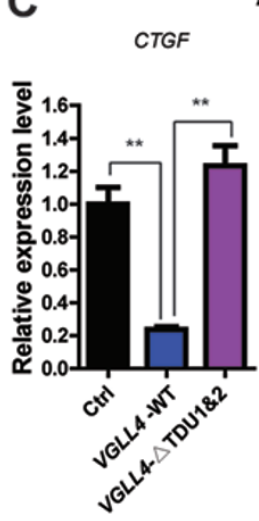

A549

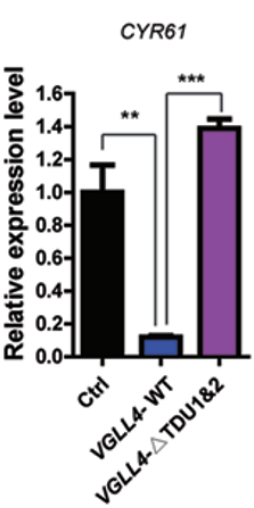

$\mathbf{F}$

CRL-5807

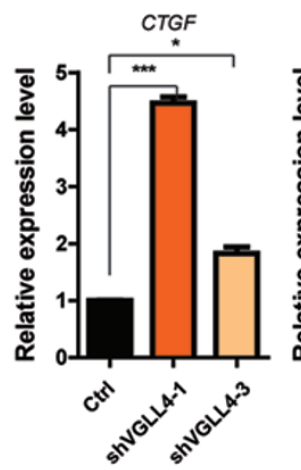

Figure 5 VGLL4 inhibits the transcriptional activity of TEADs and the expression of their downstream target genes. (A) Ectopic expression of wild-type (WT) VGLL4 but not VGLL4 mutant with deletion of two TDU domains decreases TEAD4dependent luciferase reporter activity in HEK-293T cells. Data are shown as means \pm SEM. ${ }^{* * *} P<0.005$. (B and C) Ectopic expression of WT VGLL4 but not VGLL4 mutant with deletion of two TDU domains significantly downregulates the expression of TEAD downstream target genes (CTGF and CYR61) in HEK-293T cells (B) and A549 cells (C). Data are shown as means \pm SEM. ${ }^{*} P<0.05$; ${ }^{* *} P<0.01$; ${ }^{* *} P<0.005$. (D) Knockdown of VGLL4 increases TEAD4-dependent luciferase reporter activity in HEK-293T cells. Data are shown as means \pm SEM. ${ }^{* *} P<0.01 ;{ }^{* *} P<0.005$. (E and F) Knockdown of VGLL4 upregulates the transcripts of CTGF and CYR61 in Beas2B (E) and CRL-5807 (F) cells. Data are shown as means \pm SEM. ${ }^{*} P<0.05$; ${ }^{* \star} P$ $<0.01 ;{ }^{* * *} P<0.005$. 

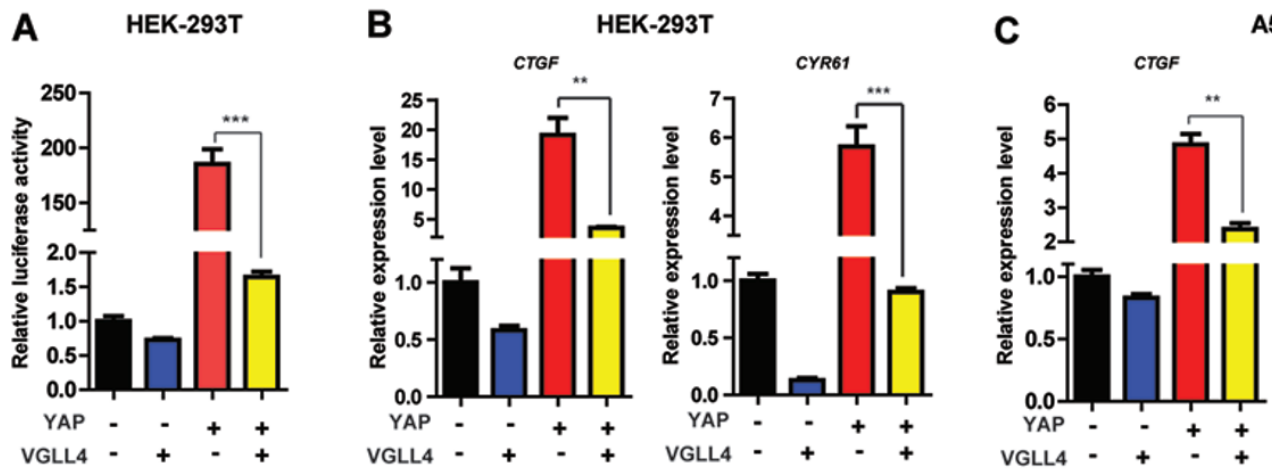

A549
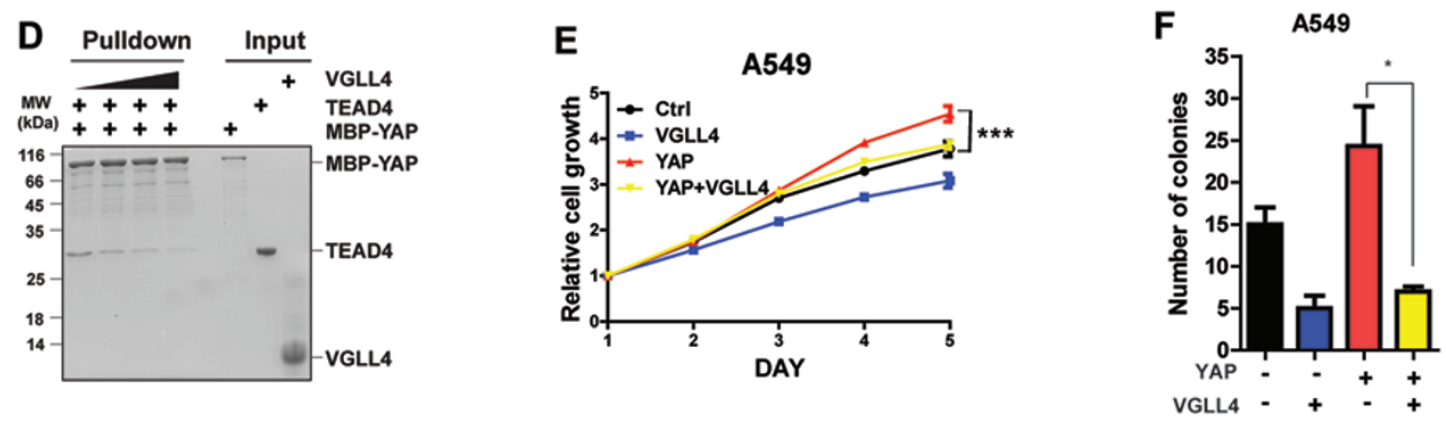

Figure 6 VGLL4 inhibits the growth-promotive function of YAP through the direct competition for its binding to TEAD4. (A) Ectopic VGLL4 expression decreases the TEAD4-dependent luciferase reporter activity promoted by YAP overexpression in HEK-293T cells. Data are shown as means \pm SEM. ${ }^{* *} P<0.005$. (B and C) Ectopic VGLL4 expression decreases YAPinduced CTGF and CYR61 expression in HEK-293T cells (B) and A549 cells (C). Data are shown as means \pm SEM. ${ }^{* *} P<$ $0.01 ;{ }^{* *} P<0.005$. (D) VGLL4 directly competes with YAP for binding to TEAD4 in vitro. YAP, amino acids 50-504 containing TEAD-binding domain. TEAD4, amino acids 217-434; VGLL4, amino acids 206-259 containing two TDU domains. (E) Ectopic VGLL4 expression significantly suppresses the growth-promotive function of YAP in A549 cells. Data are shown as means \pm SEM. ${ }^{* *} P<0.005$. (F) Ectopic expression of VGLL4 significantly inhibits the colony formation of A549 cells promoted by YAP overexpression. ${ }^{*} P<0.05$.

\section{Discussion}

In this study, we have identified VGLL4 as a potential tumor suppressor in lung cancer via negatively regulating YAP-TEAD transcriptional complex activity. Through studies of murine and human lung cancer specimens, we find that VGLL4 is significantly decreased in lung cancer. Taking advantage of lung cancer cells culture system and de novo lung cancer mouse model, we clearly showed that VGLL4 exhibits a strong inhibitory role in lung cancer growth and progression. Moreover, our data show that VGLL4 acts as a novel negative regulator of YAP-TEAD transcriptional complex through direct competition with YAP for binding to TEADs. Thus, these data have provided a novel mechanism in lung carcinogenesis through VGLL4 involving the regulation of YAP-TEAD transcription activation and Hpo pathway.

The biological function of VGLL4 was initially characterized in heart. Ectopic expression of VGLL4 in cardiac myocytes suppresses a TEF-1-dependent skeletal $\alpha$-actin promoter and interfered with its $\alpha_{1}$-adenergic induction [24]. VGLL4 is also shown to contribute to apoptosis signaling via forcing the nuclear relocation of IAPs [32]. VGLL4 was also identified as one of the mutated genes in pancreatic ADC through mutagenic screen [37]. However, the potential contribution of VGLL4 to cancer development, especially lung cancer, remains to be elucidated. Our study here provides strong evidence that VGLL4 is downregulated in a large proportion of lung cancer specimens and plays a suppressive role in lung cancer. Moreover, our data show that ectopic VGLL4 expression also significantly impairs the growth of other types of cancer cells including liver cancer cell line HepG2 as well as cervical cancer cell line Hela (Supplementary information, Figure S14). Coincidently, a recent study has convincingly shown that VGLL4 plays an inhibitory role in hepatocellular carcinoma (HCC) formation [38]. Collectively, these 
data together suggest that VGLL4 may act as a common tumor suppressor in a variety of human cancers including lung cancer and liver cancer.

VGLL4 is the only member of VGLL family that carries two TDU domains. An interesting question remains here is that which TDU domain is more important for VGLL4's suppression on lung cancer cell growth. Deletion of the second TDU domain (aa 242-252) completely abolishes the inhibitory role of VGLL4 in lung cancer cell growth while deletion of the first one (aa 214-224) does not affect VGLL4's function, indicating that the second TDU domain is more important for VGLL4's inhibitory function (Supplementary information, Figure S15). VGLL1 is another VGLL family member that has only one TDU domain. Previous studies and our own data showed that VGLL1 plays a promotive role in tumor cell growth (Supplementary information, Figure S16). Therefore, it will be interesting to further investigate how VGLL4 functions differently from other family members in relation to the TDU domain.

Studies over past decade have uncovered the vital role of Hpo pathway in organ size control and tumorigenesis [8]. Deregulation of Hpo pathway eventually triggers the transcriptional activation of the YAP-TEAD complex and initiates downstream target gene expression [39]. Both TEADs and YAP have been reported to be overexpressed or hyper-activated in various human cancers. Previous studies have demonstrated YAP as a growth promoter in both in vitro cell culture system and in vivo mouse models [40]. Recent works have also shown a critical role of YAP in stem cell self-renewal and tissue regeneration [41]. Multiple mechanisms have been proposed previously for the regulation of YAP activity. Canonical Hpo kinase cascade phosphorylates YAP at Ser 127 and leads to YAP cytoplasmic sequestration and inactivation [42]. $\alpha$-catenin acts as an upstream negative regulator of YAP through modulating its interaction with 14-3-3 and PP2A phosphatase [14]. The activity of YAP can also be modulated by protein-protein interaction and cytosol retention. For example, angiomotin (AMOT) family proteins bind to YAP and restrain YAP to tight junction and thus inhibit its nuclear translocation [34]. Moreover, Mask is proved to form complex with YAP in nucleus and is required for the full activity of YAP $[43,44]$. However, there is no endogenous negative regulator of YAP activity in the nucleus reported up to date. We here convincingly show that VGLL4, predominantly located in nucleus, inhibits YAP-driven transcriptional activity and growth advance through the competition for YAP-TEAD complex formation. Our data further showed that the two TDU domains of VGLL4 are important for this competition. Interestingly, similar findings are also observed in Drosophila.
Sd Binding Protein (SdBP)/Tgi, the Drosophila homologue of VGLL4, interacts with Sd through its TDU domains and competes with Yki in nucleus to restrict tissue growth [45]. It is worth noting that Tgi is slightly different from VGLL4 with three extra PPxY motifs, which are essential for Tgi-Yki binding. Despite the functional similarity between VGLL4 and Tgi, ectopic Tgi expression results in the formation of an inactivated Yki-TgiSd complex due to the extra PPxY motifs. In contrast, VGLL4 cannot form the complex with YAP-TEAD (data not shown). Interestingly, deletion of the PPxY motifs results in the loss of ability of Tgi in forming the Yki-TgiSd ternary complex but does not affect the competition with Yki for binding to Sd [45]. These data indicates that the competition of VGLL4 with YAP for TEADs binding is functionally and evolutionally conserved despite the different manner. Recently, Koontz et al. [38] have also demonstrated that YAP can compete with VGLL4 for binding to TEAD2. Taken together, these data show that VGLL4 and YAP compete with each other for TEAD binding and modulate downstream gene transcription and functional outcomes. As YAP acts as an oncogene in multiple human cancers and plays a crucial role in stem cell homeostasis and tissue regeneration, manipulating the interaction among YAP, TEADs and VGLL4 may provide a new entry point for human cancer treatment and regenerative medicine.

\section{Materials and Methods}

\section{Human lung cancer specimen collection}

All the human lung cancer and normal lung specimens were collected in Fudan University Shanghai Cancer Center from January 2008 to December 2009 with written consents of patients and the approval from the Institute Research Ethics Committee. A total of 30 human lung ADC samples with paired pathologically normal lungs were used for real-time PCR analysis and another 77 lung ADCs and 27 pathologically normal lungs were used for IHC analysis.

\section{Mouse cohorts, treatment and histopathological analysis}

$\operatorname{Kras}^{\mathrm{G} 12 \mathrm{D}}, L k b 1^{\mathrm{L} / \mathrm{L}}$ and $p 53^{\mathrm{L} / \mathrm{L}}$ mice were generously provided by Drs T Jacks and R Depinho. Lung cancer mouse models with $\operatorname{Kras}^{\mathrm{G} 12 \mathrm{D}}, \mathrm{p5}^{\mathrm{L} / \mathrm{L}} / \operatorname{Kras}^{\mathrm{G} 12 \mathrm{D}}, \operatorname{Lkb1}^{\mathrm{L} / \mathrm{L}} /$ Kras $^{\mathrm{G} 12 \mathrm{D}}$ mice were generated as previously described [46]. All mice were housed in a specific pathogen-free environment at the Shanghai Institute of Biochemistry and Cell Biology and treated in strict accordance with protocols approved by the Institutional Animal Care and Use Committee of the Shanghai Institutes for Biological Sciences, Chinese Academy of Sciences.

The $\operatorname{Kras}^{\mathrm{G} 12 \mathrm{D}}, p 53^{\mathrm{L} / \mathrm{L}} / \operatorname{Kras}^{\mathrm{G} 12 \mathrm{D}}, \operatorname{Lkb1}^{\mathrm{L} / \mathrm{L}} / \mathrm{Kras}^{\mathrm{G} 12 \mathrm{D}}$ mice at 6-8 weeks of age were treated with either $2 \times 10^{6}$ PFU of AdenoCre virus or lentivirus (Lenti-Cre or VGLL4-CRE) via nasal inhalation as described before [47]. The lentiviruses were prepared as previously described [33]. All mice were sacrificed for gross 
inspection and histopathological examination. Lung tumors were dissected for molecular analysis. Histopathological analysis was conducted as previously described. Briefly, mice lung tissues were inflated and fixed in $4 \%$ formalin, embedded in paraffin and sectioned for hematoxylin and eosin (H\&E) staining. Tumor number was counted under microscope and tumor size was analyzed using Image-J software.

\section{Cell culture, transfection and lentiviral infection}

Human lung ADC cell lines A549, CRL-5807, CRL-5872 were cultured in RPMI 1640 supplemented with 10\% FBS. HEK-293T, Beas2B, Hela and HepG2 were cultured in DMEM containing $10 \%$ FBS. Lentiviral infection was done as follows: HEK-293T cells were co-transfected with pLKO.1 or pCDH constructs and packaging plasmids. The progeny viruses released from HEK293 T cells were filtered, collected and used to infect A549, CRL5807, CRL-5872, Beas2B, Hela and HepG2 cells.

\section{Cellular functional assay}

Virus-infected cells were seeded in 96-well plate and the viability of cells was measured by MTT assay daily for 5 days. Briefly, $20 \mu \mathrm{l}$ of MTT working solution $(5 \mathrm{mg} / \mathrm{ml})$ was added into each well and incubated at $37{ }^{\circ} \mathrm{C}$ for $4 \mathrm{~h}$. Then the supernatants were removed and the resultant MTT formazan was dissolved in $100 \mu \mathrm{l}$ of DMSO. The absorbance was measured at the wavelengths of $570 \mathrm{~nm}$ and $630 \mathrm{~nm}$.

For cell cycle analysis, virus-infected cells with $80 \%$ confluence were harvested and fixed with $75 \%$ ethanol. Then cells were taken for PI staining and cell cycle was analyzed using flow cytometry.

For soft agar colony formation assay, virus-infected cells were added to growth medium with $0.2 \%$ agar and layered onto $1 \%$ agar beds in six-well plates. Cells were fed with $1 \mathrm{ml}$ of medium every three days. The colonies were stained with $0.005 \%$ crystal violet and counted in 2-3 weeks.

For 3D cell culture, virus-infected cells were seeded in medium containing 2\% Matrigel (BD Biosciences) on the top of another layer of solidified Matrigel. Cells were then cultured at $37^{\circ} \mathrm{C}$ for 1 week. Photos were taken using a light microscope (Leica).

\section{Luciferase reporter assay}

HEK-293T cells were seeded in 24-well plates. Luciferase reporter and the indicated plasmids were co-transfected. Luciferase activities were measured $48 \mathrm{~h}$ after transfection using DualLuciferase Assay kit (Promega) on GloMax 20/20 luminometer (Promega) following the manufacturer's instructions. pRL-TK was co-transfected as internal control. Experiments were done in triplicates.

\section{RT-PCR and quantitative real-time PCR}

Total RNA was isolated using Trizol reagent (Invitrogen) and retro-transcribed into first-strand cDNA using RevertAid ${ }^{\mathrm{TM}}$ First Strand cDNA Synthesis Kit (Fermentas). cDNAs were subjected to quantitative real-time PCR with gene-specific primers on 7500 Fast Real-Time PCR System (Applied Biosystems) using SYBRGreen Master PCR mix (Invitrogen). GAPDH (human) or actin (mouse) served as internal control.

Primers used were:

Human VGLL 4: 5'-AACTGCAACCTCTCGCACTG-3' (forward); 5'-GCTCGGGCTCCTTGTAATTCT-3' (reverse).
Human YAP: 5'-CACAGCATGTTCGAGCTCAT-3' (forward); 5'-GATGCTGAGCTGTGGGTGTA-3' (reverse).

Human $C T G F$ : 5'-CCTGGTCCAGACCACAGAGT-3' (forward); 5'-TGGAGATTTTGGGAGTACGG-3' (reverse).

Human CYR61: 5'-A CCGCTCTGAAGGGGATCT-3' (forward); 5'-ACTGATGTTTACAGTTGGGCTG-3' (reverse).

Human GAPDH: 5'-CAGGTGGTCTCCTCTGACTT-3' (forward); 5'-CCAAATTCGTTGTCATACCA-3' (reverse).

Human TEAD 1: 5'-GGCCGGGAATGATTCAAACAG-3' (forward); 5'-CAATGGAGCGACCTTGCCA-3' (reverse).

Human TEAD 3: 5'-TCATCCTGTCAGACGAGGG-3' (forward); 5'-TCTTCCGAGCTAGAACCTGTAT-3' (reverse).

Human TEAD4: 5'-GAACGGGGACCCTCCAATG-3' (forward); 5'-GCGAGCATACTCTGTCTCAAC-3' (reverse).

Mouse Actin: 5'-TGAGCGCAAGTACTCTGTGTGGAT-3' (forward); 5'-ACTCATCGTACTCCTGCTTGCTGA-3' (reverse).

Mouse $V g l l 4$ : 5'-ATGAACAACAATATCGGCGTTCT-3' (forward); 5'-GGGCTCCATGCTGAATTTCC-3' (reverse).

\section{IHC study}

IHC was performed as previously described [33]. The proliferation rate was evaluated by counting Ki-67-positive nuclear staining at high-power field (HPF) for more than 30 fields for each group. The VGLL4 immunostainning was reviewed and scored blindly. Briefly, the scoring system used in grading the level of nuclear VGLL4 expression was as follows: VGLL4 ${ }^{\text {Low }}$, nuclear expression is low or nuclear expression is less than $50 \%$ of VGLL4positive staining; VGLL4 ${ }^{\mathrm{High}}$, nuclear expression is more than $50 \%$ of VGLL4-positive staining.

\section{Western blot and immunofluorescence}

Western blot was conducted as described before [48]. For immunofluorescence, cells cultured on cover slips were fixed with $4 \%$ paraformaldehyde and permeabilized with $0.1 \%$ Triton $\mathrm{X}-100$ in $1 \times$ PBS. Cells were blocked with $3 \%$ bovine serum albumin (BSA) and incubated with primary antibody overnight at $4{ }^{\circ} \mathrm{C}$. Then cells were washed with immunofluorescence buffer and incubated with Alexa Fluor 555 or 488 conjugated secondary antibodies. Nuclei were counterstained with Hoechst. After washing with immunofluorescence buffer, cells were mounted and visualized using a laser scanning confocal microscope.

\section{Co-immunoprecipitation}

HEK-293T cells were transfected with the indicated plasmids using Lipofectamine according to the manufacturer's instructions. Cells were lysed and the supernatants were incubated with antiHA, anti-FLAG antibodies and protein A/G agarose (Santa Cruz). The immune complexes were subjected to SDS-PAGE, and analyzed by western blot.

\section{Pull-down assay}

MBP-fused YAP (aa 50-504 containing TEAD-binding domain) coupled on amylose resin were mixed with $10 \mu \mathrm{g}$ TEAD4 (aa 217434 ) in the presence of gradually increased amounts of VGLL4 (aa 206-259 containing two TDU domains) from 0 to $40 \mu \mathrm{g}$ at $4{ }^{\circ} \mathrm{C}$ for $1 \mathrm{~h}$ in $20 \mathrm{mM}$ HEPES (pH 7.5), $300 \mathrm{mM} \mathrm{NaCl}, 5 \%$ glycerol, and then washed three times. The proteins bound on the resin were eluted by the same buffer with $20 \mathrm{mM}$ maltose. The input and output samples were loaded on $15 \%$ SDS-PAGE followed by 
Coomassie Blue staining.

\section{Antibodies and plasmids}

The following antibodies were used: VGLL4, Actin, HA, FLAG (all from Sigma-Aldrich); Ki-67 (Epitomics Inc.); YAP (Santa Cruz); HRP-conjugated rabbit and mouse secondary antibodies (Santa Cruz); Alexa Fluor 555 or 488 conjugated antimouse or anti-rabbit secondary antibodies (Invitrogen).

The following plasmids were used: pCI-HA-TEAD4 was generously provided by Dr Zengqiang Yuan (Institute of Biophysics, Chinese Academy of Sciences); TEAD1/3/4 shRNA construct, CTGF promoter and pGal4-TEAD4/5xUAS-Luc reporter system were kindly given by Dr Kunliang Guan (Fudan University).

To construct the human YAP1 expression plasmid, the coding sequence of human YAP1 was amplified with primers:

F o r w ard: $5^{\prime}$ - A C C T C T A C G A A T T C G C C A C CATGGGATACCCCTACGACGTCCCCGACTACGCCGATCCCGGGCAGCAGCCGC-3'; reverse: 5'-ACCTCTACGGATCCCTATAACCATGTAAGAAAGCTTTCTTTATCTAGCTTGG-3'.

To construct the human VGLL4 expression plasmid, the coding sequence of human VGLL4 was amplified with primers:

Forward: 5'-CGGAATTCGCCACCATGCTATTTATGAAGATGGACC-3'; reverse: 5'-ATTTGCGGCCGCTCAGGAGACCACAGAGGGGGAG-3'.

To construct the human VGLL1 expression plasmid, the coding sequence of human VGLL1 was amplified with primers:

Forward: 5'-GCTCTAGAGCCACCATGGGATACCCCTACG A C G T C C C C G A C T A C G C C A T G G A A G A A A T GAAGAAGACT-3'; reverse: 5'-CGGGATCCCTAAAGATGCTGCAGGTATCGATG-3'.

The DNA fragment was inserted into pCDH vector or pCDNA3.1 vector. The plasmids pCDH-Flag-VGLL4- $\Delta$ TDU1, pCDH-Flag-VGLL4- $\Delta$ TDU2, pCDH-Flag-VGLL4- $\Delta$ TDU1\&2, pCDNA3.1-Flag-VGLL4- $\triangle$ TDU1\&2 were constructed using QuikChange ${ }^{\mathbb{B}}$ XL Site-Directed Mutagenesis Kit (Agilent Technologies). To generate the human VGLL4 knockdown construct, the following oligonucleotides were cloned into pLKO.1 at the $A g e \mathrm{I} / \mathrm{EcoRI}$ sites:

shVGLL4-1:

sense: 5'-CCGGGAGCCTGGGCAAGAATTACAACTCGAGTTGTAATTCTTGCCCAGGCTCTTTTTG-3'; antisense: 5'-AATTCAAAAAGAGCCTGGGCAAGAATTACAACTCGAGTTGTAATTCTTGCCCAGGCTC-3'.

shVGLL4-2

sense: 5'-CCGGCATCTGAACAAGACTGCCAATCTCGAGATTGGCAGTCTTGTTCAGATGTTTTTG-3'; antisense: 5'-AATTCAAAAACATCTGAACAAGACTGCCAATCTCGAGATTGGCAGTCTTGTTCAGATG-3'.

shVGLL4-3:

sense: 5'-CCGGCAGGAGCCTGGGCAAGAATTACTCGAGTAATTCTTGCCCAGGCTCCTGTTTTTG-3'; antisense: 5'-AATTCAAAAACAGGAGCCTGGGCAAGAATTACTCGAGTAATTCTTGCCCAGGCTCCTG-3'.

For pull-down assay, YAP (aa 50-504) was inserted into pET28a with MBP tag, and VGLL4 (aa 206-259) and TEAD4 (aa 217-434) were inserted into pET28a with His-SUMO tag.

\section{Statistical analysis}

All statistical analyses were carried out using the SPSS 13.0 statistical software package. Data were analyzed by Student's $t$ test (two tailed). $P<0.05$ was considered to be significant. Error bars represent SEM.

\section{Acknowledgments}

We thank Drs T Jacks, R Depinho, K Wong for providing the $\operatorname{Kras}^{\mathrm{G} 12 \mathrm{D}}, L k b 1^{\mathrm{L} / \mathrm{L}}$ and $p 53^{\mathrm{L} / \mathrm{L}}$ mice; Drs Kunliang Guan, Bin Zhao, Qunying Lei, Zengqiang Yuan for sharing reagents; Drs Dangsheng $\mathrm{Li}$, Yun Zhao for invaluable comments on the manuscript and Drs Yan Ren, Zhaoyuan Fang, Bin Gao, Yihua Sun, Junhua Zhang for technical supports. This work was supported by Ministry of Science and Technology of China (2012CB910800, 2010CB912102, 2012CB945001), the Cross and Cooperation in Science and Technology Innovation Team program, the Strategic Priority Research Program of the Chinese Academy of Sciences (XDA01010406), the National Natural Science Foundation of China (31370747), Post-Doctor Research Program of Shanghai Institutes for Biological Sciences (2012KIP502, 2013KIP303, 2012KIP401), China Postdoctoral Science Foundation (2011M500826, 2013T60476), the Sanofi-Aventis Shanghai Institutes for Biological Sciences (SA-SIBS) scholarship program and KC Wong Education Foundation Scholarship. The authors gratefully acknowledge the support of SA-SIBS scholarship.

\section{References}

1 Siegel R, Naishadham D, Jemal A. Cancer statistics, 2012. $C A$ Cancer J Clin 2012; 62:10-29.

2 Jackson EL, Willis N, Mercer K, et al. Analysis of lung tumor initiation and progression using conditional expression of oncogenic K-ras. Genes Dev 2001; 15:3243-3248.

3 Tuveson DA, Jacks T. Modeling human lung cancer in mice: similarities and shortcomings. Oncogene 1999; 18:5318-5324.

4 Larsen JE, Minna JD. Molecular biology of lung cancer: clinical implications. Clin Chest Med 2011; 32:703-740.

5 Zhao B, Lei QY, Guan KL. The Hippo-YAP pathway: new connections between regulation of organ size and cancer. Curr Opin Cell Biol 2008; 20:638-646.

6 Pan D. Hippo signaling in organ size control. Genes Dev 2007; 21:886-897.

7 Yin M, Zhang L. Hippo signaling: a hub of growth control, tumor suppression and pluripotency maintenance. $J$ Genet Genomics 2011; 38:471-481.

8 Pan D. The hippo signaling pathway in development and cancer. Dev Cell 2010; 19:491-505.

9 Steinhardt AA, Gayyed MF, Klein AP, et al. Expression of Yes-associated protein in common solid tumors. Hum Pathol 2008; 39:1582-1589.

10 Wang Y, Dong Q, Zhang Q, Li Z, Wang E, Qiu X. Overexpression of yes-associated protein contributes to progression and poor prognosis of non-small-cell lung cancer. Cancer Sci 2010; 101:1279-1285.

$11 \mathrm{Xu} \mathrm{MZ,} \mathrm{Yao} \mathrm{TJ,} \mathrm{Lee} \mathrm{NP,} \mathrm{et} \mathrm{al.} \mathrm{Yes-associated} \mathrm{protein} \mathrm{is} \mathrm{an}$ independent prognostic marker in hepatocellular carcinoma. Cancer 2009; 115:4576-4585.

12 Zhang X, George J, Deb S, et al. The Hippo pathway tran- 
scriptional co-activator, YAP, is an ovarian cancer oncogene. Oncogene 2011; 30:2810-2822.

13 Zhao B, Ye X, Yu J, et al. TEAD mediates YAP-dependent gene induction and growth control. Genes Dev 2008; 22:19621971.

14 Schlegelmilch K, Mohseni M, Kirak O, et al. Yap1 acts downstream of alpha-catenin to control epidermal proliferation. Cell 2011; 144:782-795.

15 Dong J, Feldmann G, Huang J, et al. Elucidation of a universal size-control mechanism in Drosophila and mammals. Cell 2007; 130:1120-1133.

16 Song H, Mak KK, Topol L, et al. Mammalian Mst1 and Mst2 kinases play essential roles in organ size control and tumor suppression. Proc Natl Acad Sci USA 2010; 107:1431-1436.

17 Zhou D, Conrad C, Xia F, et al. Mst1 and Mst2 maintain hepatocyte quiescence and suppress hepatocellular carcinoma development through inactivation of the Yap1 oncogene. Cancer Cell 2009; 16:425-438.

18 Anbanandam A, Albarado DC, Nguyen CT, Halder G, Gao X, Veeraraghavan S. Insights into transcription enhancer factor 1 (TEF-1) activity from the solution structure of the TEA domain. Proc Natl Acad Sci USA 2006; 103:17225-17230.

19 Knight JF, Shepherd CJ, Rizzo S, et al. TEAD1 and c-Cbl are novel prostate basal cell markers that correlate with poor clinical outcome in prostate cancer. Br J Cancer 2008; 99:1849-1858.

20 Adelaide J, Finetti P, Bekhouche I, et al. Integrated profiling of basal and luminal breast cancers. Cancer Res 2007; 67:11565-11575.

21 Nowee ME, Snijders AM, Rockx DA, et al. DNA profiling of primary serous ovarian and fallopian tube carcinomas with array comparative genomic hybridization and multiplex ligation-dependent probe amplification. J Pathol 2007; 213:46-55.

22 Skotheim RI, Autio R, Lind GE, et al. Novel genomic aberrations in testicular germ cell tumors by array-CGH, and associated gene expression changes. Cell Oncol 2006; 28:315326.

23 Chan SW, Lim CJ, Loo LS, Chong YF, Huang C, Hong W. TEADs mediate nuclear retention of TAZ to promote oncogenic transformation. J Biol Chem 2009; 284:1434714358.

24 Chen HH, Mullett SJ, Stewart AF. Vgl-4, a novel member of the vestigial-like family of transcription cofactors, regulates alpha1-adrenergic activation of gene expression in cardiac myocytes. J Biol Chem 2004; 279:30800-30806.

25 Mielcarek M, Gunther S, Kruger M, Braun T. VITO-1, a novel vestigial related protein is predominantly expressed in the skeletal muscle lineage. Gene Expr Patterns 2002; 2:305310.

26 Mielcarek M, Piotrowska I, Schneider A, Gunther S, Braun T. VITO-2, a new SID domain protein, is expressed in the myogenic lineage during early mouse embryonic development. Gene Expr Patterns 2009; 9:129-137.

27 Pobbati AV, Hong W. Emerging roles of TEAD transcription factors and its coactivators in cancers. Cancer Biol Ther 2013; 14:390-398.

28 Vaudin P, Delanoue R, Davidson I, Silber J, Zider A. TONDU (TDU), a novel human protein related to the product of vestigial (vg) gene of Drosophila melanogaster interacts with vertebrate TEF factors and substitutes for Vg function in wing formation. Development 1999; 126:4807-4816.

29 Pobbati AV, Chan SW, Lee I, Song H, Hong W. Structural and functional similarity between the Vgll1-TEAD and the YAPTEAD complexes. Structure 2012; 20:1135-1140.

30 Richardson AL, Wang ZC, De Nicolo A, et al. X chromosomal abnormalities in basal-like human breast cancer. Cancer Cell 2006; 9:121-132.

31 Helias-Rodzewicz Z, Perot G, Chibon F, et al. YAP1 and VGLL3, encoding two cofactors of TEAD transcription factors, are amplified and overexpressed in a subset of soft tissue sarcomas. Genes Chromosomes Cancer 2010; 49:11611171.

32 Jin HS, Park HS, Shin JH, et al. A novel inhibitor of apoptosis protein (IAP)-interacting protein, Vestigial-like (Vgl)-4, counteracts apoptosis-inhibitory function of IAPs by nuclear sequestration. Biochem Biophys Res Commun 2011; 412:454459.

33 Feng Y, Wang Y, Wang Z, et al. The CRTC1-NEDD9 signaling axis mediates lung cancer progression caused by LKB1 loss. Cancer Res 2012; 72:6502-6511.

34 Zhao B, Li L, Lu Q, et al. Angiomotin is a novel Hippo pathway component that inhibits YAP oncoprotein. Genes Dev 2011; 25:51-63.

35 Zhang H, Pasolli HA, Fuchs E. Yes-associated protein (YAP) transcriptional coactivator functions in balancing growth and differentiation in skin. Proc Natl Acad Sci USA 2011; 108:2270-2275.

36 Muramatsu T, Imoto I, Matsui T, et al. YAP is a candidate oncogene for esophageal squamous cell carcinoma. Carcinogenesis 2011; 32:389-398.

37 Mann KM, Ward JM, Yew CC, et al. Sleeping Beauty mutagenesis reveals cooperating mutations and pathways in pancreatic adenocarcinoma. Proc Natl Acad Sci USA 2012; 109:5934-5941.

38 Koontz LM, Liu-Chittenden Y, Yin F, et al. The Hippo effector Yorkie controls normal tissue growth by antagonizing scalloped-mediated default repression. Dev Cell 2013; 25:388-401.

39 Zhao B, Li L, Guan KL. Hippo signaling at a glance. J Cell Sci 2010; 123:4001-4006.

40 Hong W, Guan KL. The YAP and TAZ transcription coactivators: key downstream effectors of the mammalian Hippo pathway. Semin Cell Dev Biol 2012; 23:785-793.

41 Zhao B, Tumaneng K, Guan KL. The Hippo pathway in organ size control, tissue regeneration and stem cell self-renewal. Nat Cell Biol 2011; 13:877-883.

42 Zhao B, Wei X, Li W, et al. Inactivation of YAP oncoprotein by the Hippo pathway is involved in cell contact inhibition and tissue growth control. Genes Dev 2007; 21:2747-2761.

43 Sansores-Garcia L, Atkins M, Moya IM, et al. Mask is required for the activity of the Hippo pathway effector Yki/ YAP. Curr Biol 2013; 23:229-235.

44 Sidor CM, Brain R, Thompson BJ. Mask proteins are cofactors of Yorkie/YAP in the Hippo pathway. Curr Biol 2013; 23:223-228.

45 Guo T, Lu Y, Li P, et al. A novel partner of Scalloped regulates Hippo signaling via antagonizing Scalloped-Yorkie activity. 
Cell Res 2013. doi: 10.1038/cr.2013.120.

46 Ji H, Ramsey MR, Hayes DN, et al. LKB1 modulates lung cancer differentiation and metastasis. Nature 2007; 448:807810.

47 DuPage M, Dooley AL, Jacks T. Conditional mouse lung cancer models using adenoviral or lentiviral delivery of Cre recombinase. Nat Protoc 2009; 4:1064-1072.

48 Gao Y, Xiao Q, Ma H, et al. LKB1 inhibits lung cancer progression through lysyl oxidase and extracellular matrix remodeling. Proc Natl Acad Sci USA 2010; 107:18892-18897.

(Supplementary information is linked to the online version of the paper on the Cell Research website.) 